Unsere Experten beraten Sie bei kniffeligen medizinischen Fragestellungen aus Ihrer Praxis.
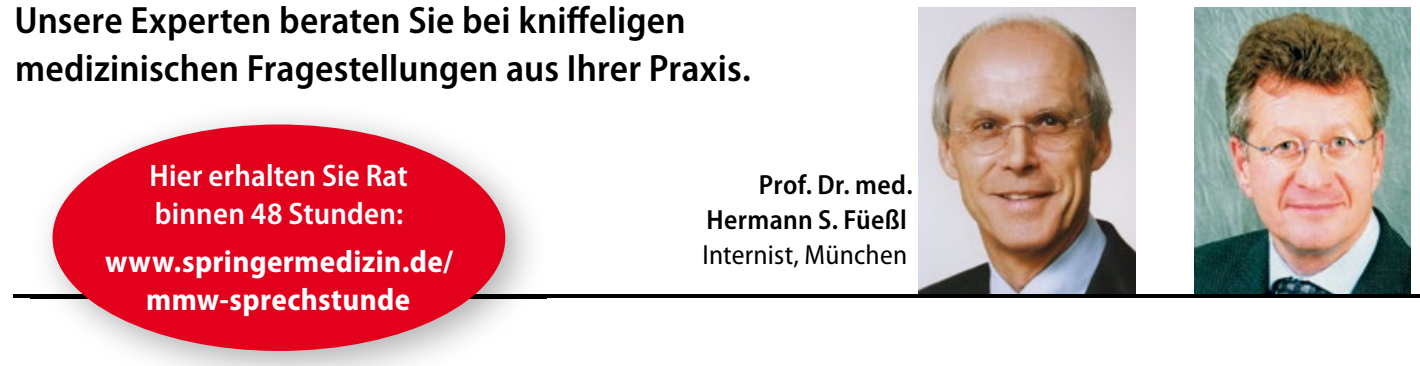

Dr. med.

Peter Stiefelhagen

Internist, Hachenburg

\title{
Idiopathische Schmerzen
}

\section{Warum tun bei Kälte die Schenkel weh?}

\begin{abstract}
? Thomas Krause, Reutlingen: Meine Patientin, eine 21 Jahre alte Krankenschwester, beklagt seit drei Jahren ziehende Schmerzen in beiden Oberschenkeln, abgeschwächt auch in den Armen und teilweise im Gesicht. Die Beschwerden treten nur bei Temperaturen $<15^{\circ} \mathrm{C}$ auf. Hautveränderungen wie Hyperämie oder Urtikaria bestehen nicht. Bei der Großmutter seien in höherem Alter ähnliche Beschwerden aufgetreten. Meine Patientin erhielt initial Amineurin $10 \mathrm{mg} / \mathrm{d}$, im Verlauf mussten wir auf $25 \mathrm{mg} / \mathrm{d}$ steigern. Ohne Medikament erreichen die Schmerzen 7-8 Punkte auf der visuellen Analogskala, unter Amineurin sind es nur 2-3 Punkte.

Folgende Diagnostik blieb ohne Befund: CMRT (Ausschluss multiple Sklerose), MRT-LWS+Becken, Abdomen-
\end{abstract}

Physiotherapie hilft manchen Patienten mit Small-fibreNeuropathie.

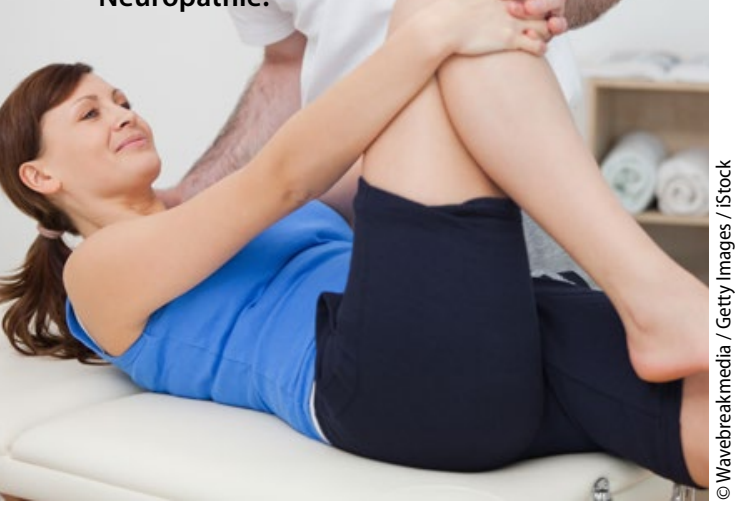

Sonografie, Gastroskopie (Ausschluss Malabsorptionsstörung), angiologische Sonografie der Becken-Bein-Gefäße und Arme, Nervenleitgeschwindigkeiten, mehrfache Konsultationen bei Neurologen, Rheumatologen, Dermatologen, Gynäkologen.

Laborchemisch fallen lediglich grenzwertige Ferritin- und Vitamin-DSpiegel auf. Eine Substitution haben wir begonnen. Insbesondere die Werte von Vitamin $B_{12}, H b A_{1 c}$, Diff-BB, ANA, ANCA, RF, BSG, CRP und Schilddrüse sind unauffällig.

Ich denke dennoch an eine neurologische Erkrankung, z. B. eine Smallfibre-Neuropathie. Halten Sie eine Probebiopsie für indiziert? Oder wäre z. B. eine Prednisolon-Therapie ex juvantibus indiziert?

! MMW-Experte Stiefelhagen: Meine Güte, da ist ja schon alles gemacht worden, was zwar gut, aber auch teuer und letztendlich nicht zielführend war. Mir fällt beim besten Willen nichts ein, was man sinnvollerweise noch machen könnte. Die Indikation für eine Nervenbiopsie sollte ein Neurologe stellen. Von einer Ex-juvantibus-Therapie mit Kortison ohne Diagnose halte ich nichts. Es spricht doch alles dafür, dass es sich um eine somatoforme Störung handelt.

MMW-Experte FüeßI: Ihrer Schilderung nach kann es sich nicht um ein lokales Problem handeln, sondern es muss eine systemische Störung vorliegen. Eine metabolisch oder paraneoplas- tisch bedingte Neuropathie ist aufgrund Ihrer Untersuchungen mit größter Wahrscheinlichkeit ausgeschlossen. Trotz des negativen MRT-Befunds ist eine beginnende multiple Sklerose immer noch möglich.

Die Symptomatik würde auch gut zu einer Small-fibre-Neuropathie passen. Für diese Diagnose wäre eine Hautbiopsie erforderlich. Übliche elektrophysiologische Methoden wie NLG und EMG lassen einen hier im Stich. Die Therapie wäre aber auch bei diagnostischer Sicherheit nur symptomatisch - und müsste zudem nach der Trial-and-ErrorMethode festgelegt werden.

In Betracht kommen Kalziumkanalmodulierende Antikonvulsiva wie Pregabalin oder Gabapentin, Natriumkanalblocker wie Carbamazepin oder Lamotrigin, trizyklische Antidepressiva wie Amitriptylin und selektive SNRI wie Duloxetin oder Venlafaxin. Häufig muss man zwei oder drei Wirkstoffe aus diesen Klassen kombinieren. Erfolgsparameter ist die klinische Wirksamkeit, laborchemische oder elektrophysiologische Kontrollparameter existieren nicht. Zudem kann die Effektivität leider immer erst nach mehreren Wochen beurteilt werden.

Topische Therapieoptionen sind Lidocain oder Capsaicin, die keine systemischen Nebenwirkungen aufweisen. Auch nicht-medikamentöse Maßnahmen wie die transkutane elektrische Nervenstimulation, Physio- und Ergotherapie sowie Psychotherapie könnten erfolgversprechend sein. 